\title{
Le travail des ingénieurs en entreprises
}

Formation, socialisation et activités des ingénieurs en perspective européenne de longue durée

Engineers at work in companies. Training, socialization and engineering activities in long-term European perspective

\section{Florent Le Bot et Alain P. Michel}

\section{OpenEdition}

\section{Journals}

Édition électronique

URL : https://journals.openedition.org/artefact/6208

DOI : 10.4000/artefact.6208

ISSN : 2606-9245

Éditeur :

Association Artefact. Techniques histoire et sciences humaines, Presses universitaires du Midi

Édition imprimée

Date de publication : 7 janvier 2021

Pagination : $9-46$

ISBN : 978-2-8107-0706-5

ISSN : 2273-0753

\section{Référence électronique}

Florent Le Bot et Alain P. Michel, « Le travail des ingénieurs en entreprises », Artefact [En ligne], 13|

2020, mis en ligne le 23 décembre 2020, consulté le 21 septembre 2021. URL : http://

journals.openedition.org/artefact/6208; DOI : https://doi.org/10.4000/artefact.6208

\section{(c) (†) $\ominus$}

Artefact, Techniques, histoire et sciences humaines est mise à disposition selon les termes de la Licence Creative Commons Attribution - Pas d'Utilisation Commerciale - Pas de Modification 4.0 International. 


\title{
Le travail des ingénieurs en entreprises
}

\author{
Formation, socialisation et activités des ingénieurs \\ en perspective européenne de longue durée
}

\author{
Florent Le Bot et Alain P. Michel
}

\section{Résumé}

Peut-on dégager une typologie des ingénieurs en Europe occidentale selon leur parcours de formation, leur mode de socialisation et leur intégration dans les entreprises ? Cette interrogation entre en résonance avec la nouvelle question d'histoire contemporaine pour les concours d'enseignement : " Le travail en Europe occidentale des années 1830 aux années 1930. Mains-d'œuvre artisanales et industrielles, pratiques et questions sociales ». Dans cet article, nous relions dans un premier temps ingénieurs et processus d'industrialisation. Puis nous corrélons la structuration des systèmes de formation aux configurations étatiques de l'ouest européen. Nous étudions ensuite les modalités de socialisation des ingénieurs et abordons les enjeux de la féminisation des mondes d'ingénieur. Enfin, nous nous intéressons aux ingénieurs en action et dans les entreprises. L'ingénieur est un personnage à facettes multiples. Son portrait et celui de son groupe, change au gré des transformations du paysage dans lequel il interagit. Nous nous efforçons d’en dégager de grandes caractéristiques.

\section{Mots-clés}

formation, industrialisation, innovation, socialisation, travail

95 Florent Le Bot et Alain P. Michel, « Le travail des ingénieurs en entreprises. Formation, socialisation et activités des ingénieurs en perspective européenne de longue durée », Artefact, 13, 2020, p. 9-46. 


\section{Engineers at work in companies. Training, socialization and engineering activities in long-term European perspective}

\section{Abstract}

Engineers work. Who are they? How were they trained? What are they doing? For which companies and under what conditions? Can we identify a typology of engineers in Western Europe of the $x \mathrm{X}^{\text {th }}-\mathrm{Xx}{ }^{\text {th }}$ century? It is not easy to answer these interrogations which resonate with the new subject of contemporary history for teaching competitions in France: "Work in Western Europe from the 1830 s to the 1930s. Hands craft and industrial work, practices and social issues". This opening paper has been informed by this specific focus. First, we link the need for new engineers with the industrialization processes. Then we correlate the structuring of training systems with the specific state configurations. Next we study the ways of socialization of engineers and tackle the challenges of the feminization of the engineering profession. Finally, we study a few cases of engineers in action in the companies they work for. An engineer is a multifaceted character. His portrait and that of his group, changes according to the transformations of the landscape in which he interacts. We strive to bring out the major features of European engineers.

\section{Keywords}

firm, training, industrialization, innovation, engineers, socialization, labour 


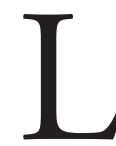

es ingénieurs travaillent.

Qui sont-ils et comment ont-ils été formés ? Que font-ils et dans quelles conditions? Pour quelles entreprises? Quelles différences et quels points communs entre les pays de l'Europe occidentale?

Il n'est pas aisé de répondre à ces questions tandis qu'elles entrent en résonance avec la nouvelle question d'histoire contemporaine pour les concours d'enseignement : "Le travail en Europe occidentale des années 1830 aux années 1930. Mains-d'œuvre artisanales et industrielles, pratiques et questions sociales ». Le présent dossier et son article d'ouverture ont été orientés par cette actualité. Jusque dans les années 1980, André Thépot note que, " au-delà des clichés et des jugements superficiels, les ingénieurs constituent une catégorie socio-professionnelle assez mal connue ${ }^{1}$ ». Aux grands corps d'État de l'époque moderne s'ajoute au XIX ${ }^{\mathrm{e}}$ siècle une nouvelle catégorie d'ingénieurs civils. Les premiers œuvrent au bon fonctionnement des équipements militaires ou fonctionnels de l'État. Les seconds répondent à de nouveaux besoins de l'économie en phases d'industrialisation : ils ont constamment dû adapter leurs actions aux bouleversements techniques du monde contemporain.

L'ingénieur est un personnage à facettes multiples. Son portrait change au gré des transformations du paysage dans lequel il interagit. L'arrièreplan est celui d'une fascination positiviste pour la technique susceptible de répondre à tous les problèmes et de pourvoir à l'ensemble des besoins de l'humanité : un imaginaire techniciste, tel que présenté et interrogé dans les travaux de François Jarrige par exemple ${ }^{2}$. Les représentations culturelles sont une source parmi d'autres. L'historiographie en général, celle des techniques en particulier, les a mobilisées dans le contexte de ce que l'on a appelé le cultural turn ${ }^{3}$. Elle souligne la mixité de la culture technique des ingénieurs avec celles des autres groupes sociaux qu'ils côtoient dans un même milieu industriel : ouvriers, techniciens, contremaîtres, directeurs, entrepreneurs ${ }^{4}$. Cette position apparemment intermédiaire entre patronat et classe ouvrière n'aide pas à leur identification à mesure que leurs fonctions se diversifient et que leur nombre augmente.

1. Thépot, 1985 , p. 9.

2. Jarrige, 2014.

3. Edgerton, 2013.

4. Kolboom, 1982 ; Moutet, 1984 ; Cohen, 1988 ; Schweitzer-Vandecasteele, 1990. 
Ainsi, le lexique des représentations ne suffit pas à couvrir l'ensemble des activités concrètes et à rendre compte de la réalité du travail des ingénieurs, ni à suivre le processus de professionnalisation d'un groupe social hybride en transformation. À l'inverse, le fait de replacer des activités techniques dans leur contexte - politique, économique et social - n'en garantit pas seul l'historicité. De ce fait, si l'émergence d'un nouveau groupe professionnel dans le monde du travail industriel est manifeste, son histoire reste compliquée à cerner. Comment rendre compte historiquement d'un groupe d'experts aux fonctions en cours de métamorphose ? Quelles perspectives choisir et quelles sources mobiliser?

Au-delà de ce qui est dit, écrit et montré des ingénieurs, Liliane HilairePérez et ses collègues recommandent de confronter les sources disponibles aux traces des " épreuves du terrain ", de replacer les ingénieurs dans le cadre des chantiers sur lesquels ils travaillent, là où ils affrontent des résistances et où ils doivent faire des compromis pour aboutir. Or, "selon les périodes et les lieux le processus de professionnalisation se traduit par une série de décalages ${ }^{6} »$. Tout ne se passe pas de la même façon partout en Europe et, pour dépasser un vieux débat, cela ne se pose pas simplement en termes d'avance ou de retards.

L'objectif de ce dossier est donc de saisir les ingénieurs dans leurs pratiques, à travers les institutions qu'ils investissent et à partir des entreprises dans lesquelles ils exercent. C'est dans ce cadre qu'ils publient leurs réflexions, qu'ils produisent des archives et qu'ils laissent des traces de leurs activités. La nécessité d'un ajustement récurrent de leurs pratiques est au cœur des préoccupations des écoles qui les forment, des firmes qui les emploient et des réseaux qui les fédèrent. Les sources sont nombreuses parce que les ingénieurs écrivent (plus que les ouvriers), mais dans un cadre contraint et réservé du fait de leur position intermédiaire entre la direction et le reste de la main-d'œuvre. Quelle est la nature de ces archives? Quelles publications? Quelles images ? Quels lieux en ont gardé la mémoire ?

Après avoir remis en perspective les métamorphoses des ingénieurs au regard des transformations des processus productifs du XIX ${ }^{e}$ au XXI ${ }^{e}$ siècle en Europe (1), nous retravaillerons la question à travers la structuration des systèmes de formation (2), qui n'est pas sans relation et sans effet sur les

5. Garçon, 2012, p. 33.

6. Blond, Hilaire-Perez et al., 2020. 
formes de socialisation des groupes d'ingénieurs (3) et les terrains de leur action en entreprises (4).

\section{Ingénieurs et processus d'industrialisation}

\section{De l'habileté des ingénieurs face à la montée en complexité des économies européennes}

Partout en Europe dans la longue durée, le monde des ingénieurs est transformé, le métier est métamorphosé, le groupe change de nature et se scinde. Demeure la distinction entre les ingénieurs d'État, qui continuent de répondre aux besoins d'une administration publique en expansion ${ }^{7}$, et les ingénieurs civils. Leurs domaines d'expertises oscillent autour des trois qualités fondamentales identifiées par Hélène Vérin ${ }^{8}$. Par leur ingéniosité, les ingénieurs sont des médiateurs rusés entre la science et les arts mécaniques. Par leur capacité à conduire des engins - d'abord machines de guerre puis outils et équipements de production -, ils sont les praticiens des activités de fabrication. Enfin, en tant que représentants du génie technique, les ingénieurs opèrent le lien entre conception, fonctionnement et optimisation de la production entreprise.

L'ingénieur "ingénieux » devient la figure centrale de l'entrepreneur-innovateur célébré au début du $\mathrm{Xx}^{\mathrm{e}}$ siècle par Schumpeter'. Jusqu'à la seconde moitié du XIX ${ }^{\mathrm{e}}$ siècle, l'innovation est majoritairement le fait d'inventeurs indépendants. En Angleterre, ils sont d'ailleurs rarement issus d'écoles d'ingénieurs ou d'universités. Ce sont des praticiens qui identifient des problèmes techniques, améliorent un dispositif et déposent des brevets qu'ils tentent de monétiser sur le marché de la propriété industrielle.

\footnotetext{
7. Même si les ingénieurs des Mines peuvent être en détachement auprès d'industriels pendant des années (Thépot, 1998). On voit également les ingénieurs des Poudres suivre la création de l'Office national de l'azote entre les deux guerres (Llopart, 2020). C'est dire que le monde des ingénieurs d'Etat n'est pas clos par rapport à l'économie industrielle. Voir aussi le rôle des ingénieurs des Mines dans le développement de l'industrie nucléaire française durant le second $\mathrm{xx}^{\mathrm{e}}$ siècle.

8. Vérin, 1993.

9. Schumpeter, 1911 et 1926 ; Bordes et al., 2011.
} 
Dans le contexte de transition de la première à la seconde industrialisation, le management de l'innovation change progressivement de forme ${ }^{10}$. Dans un premier temps, quelques pionniers du secteur automobile, le plus souvent ingénieurs, s'appuient sur leur innovation pour fonder leur propre entreprise. Rapidement, le coût d'entrée dans l'activité industrielle à forte intensité capitalistique ne permet plus ce type d'opération. L'innovation devient le produit d'un travail collectif développé au sein d'un nombre croissant de laboratoires internes et de services de recherches et développements $(\mathrm{R} \& \mathrm{D})^{11}$. Les entreprises de pointe (aluminium, électricité, etc.) conçoivent leurs propres programmes de recherche et recrutent des ingénieurs, chimistes et physiciens appointés. Dans les secteurs moins concentrés (céramique, papeterie, tannerie, etc.), des laboratoires d'essais portés par les organisations patronales, soutenus par les pouvoirs publics et souvent adossés aux écoles spécialisées d'ingénieurs, se déploient en Allemagne, en Belgique, en Grande-Bretagne, en France, etc. durant le second $\mathrm{XIX}^{\mathrm{e}}$ siècle ${ }^{12}$. Mobilisant les ressources du laboratoire, l'ingénieur-chercheur devient un "travailleur salarié de l'innovation ${ }^{13}$ ». Il se démarque de l'ingénieur d'atelier qui demeure un homme du terrain et de 14 la pratique.

\section{Les ingénieurs en quête de reconnaissance durant l'entre-deux-guerres}

Fort de cette dynamique, les ingénieurs européens engagent un autre défi : celui des méthodes d'organisation dites "scientifiques ». Si le modèle industriel américain de Frederick W. Taylor s'installe comme une référence, il n'est ni le seul, ni le meilleur, ni surtout celui qui s'adapte à toutes les situations ${ }^{14}$. Ainsi, à la suite des expériences de la Grande Guerre, Henri Fayol publie une série d'ouvrages sur l'administration de l'économie et des entreprises ${ }^{15}$. Les enjeux de la technique se déplacent vers les questions d'organisation de la production et du travail. La tendance de l'époque est celle d'une spécialisation des métiers d'ingénieurs, segmentation propice à

10. Lécuyer, 2015.

11. Fox et Guagnini, 1999.

12. Le Bot, en cours ; Brion, 1986, p. 258.

13. Boisard, Didry et al., 2016.

14. Vatin, 1990.

15. Bertilorenzi et al. (dir.), 2019. 
la sous-traitance des expertises demandées qui suscite le développement de cabinet de conseils ${ }^{16}$.

L'expertise offre un débouché pour des ingénieurs-consultants qui donnent leurs avis sur la marche des entreprises - souvent selon des modèles préconçus. Après la Première Guerre mondiale, dans l'orbite d'une Société des Nations (SDN) dont les principes fondateurs peinent à s'imposer, la création du Bureau International du travail (BIT) s'inscrit dans le cadre d'une volonté utopique d'organiser rationnellement le monde ${ }^{17}$. La première enquête lancée en 1924 par le BIT sur les travailleurs intellectuels est précisément consacrée à une vaste réflexion sur le rôle des ingénieurs, leurs conditions d'emploi, les modalités à mettre en ouvre pour faciliter leur libre circulation et leur installation dans des pays étrangers ${ }^{18}$. Les rapports révèlent une convergence des préoccupations en Europe concernant les conditions d'accès au titre d'ingénieur, en particulier pour les autodidactes, et déjà, derrière ces inquiétudes, pointe la crainte du chômage ${ }^{19}$.

Dans le sillage du BIT émerge un faisceau d'organismes déployant des expertises à la fois complémentaires et concurrentes, dans une période d'expérimentation de nouvelles formes d'organisation du travail, et tandis que se multiplient les ouvrages sur le thème de la « rationalisation ${ }^{20}$ ». Ces institutions internationales affichent des visées communes qui masquent toutefois des visions différentes, voire antagonistes, du monde de la production et du travail. Leurs discours se déploient dans le cadre de controverses larvées, pris dans les tensions liées à la menace révolutionnaire soviétique, au défi industriel américain, à l'ancrage du fascisme italien et au danger d'une renaissance de la puissance allemande à travers le nazisme.

La crise des années 1930 déclenche partout en Europe un esprit corporatiste conduisant à des mesures législatives malthusiennes. Les moyens employés s'avèrent différents selon les États, mais ils visent partout un même but : restreindre l'accès, sinon à la profession, du moins au titre. Cela se fait précocement en Italie, à l'occasion de la loi Gentile (1923); en Angleterre, c'est l'octroi de la Charte pour les sociétés d'ingénieurs (1930);

16. Henry, 2012.

17. Lespinet-Moret, 2006 ; Cayet, 2010, p. 16.

18. BIT, 1924.

19. Grelon, 1986, p. 19-20 ; Hugot-Piron, 2014.

20. Moutet, 1997. 
en France, c'est la loi du 10 août 1932 (mais le titre d'ingénieur diplômé n'est protégé qu'à l'occasion de celle du 10 juillet 1934); en Belgique, la loi du 11 septembre 1933 ; en Espagne, le décret du 14 mars 1933²1. Partout des modalités législatives ou administratives entraînent un repli sur soi. Ainsi, des mesures sont prises qui limitent la possibilité pour les entreprises d'embaucher des ingénieurs autres que les nationaux.

\section{La montée des cadres}

Chemin faisant, le rôle de l'ingénieur glisse d'une fonction essentiellement technique à la gestion du « facteur humain ». Dans la période de croissance retrouvée et de tensions sociales des années 1890, les employeurs industriels exigent d'eux qu'ils soient aussi des chefs ${ }^{22}$. L'ingénieur ne se substitue pas aux contremaîtres. Il y a une modulation des conceptions du commandement et de l'autorité dans les domaines de l'armée, qui contamine à la fois la politique et l'usine. C'est l'époque du paternalisme réformateur de Frédéric Le Play, de la doctrine sociale de l'Église et de la définition du « rôle social de l'officier " par le maréchal Hubert Lyautey. Au tournant des $\mathrm{XIX}^{\mathrm{e}}$ et $\mathrm{XX}^{\mathrm{e}}$ siècles, plusieurs textes interrogent la place de l'ingénieur dans la société. Celui d'Emile Cheysson est un modèle de plaidoyer leplaysien en faveur de la paix sociale, visant à unir deux sociétés d'ingénieurs autour d'un même objectif " libéral » : celui d'éviter que l'État n'intervienne dans les affaires privées des entreprises. "On s'endort libéral et on se réveille étatiste ${ }^{23}$. " Ce combat est repris trente-cinq ans plus tard par Georges Lamirand, chantre du " catholicisme social ${ }^{24}$ ", dans son ouvrage justement intitulé Le rôle social de l'ingénieur, avec une double préface de Lyautey et du directeur de l'École centrale ${ }^{25}$. Les usines ont grandi, les ouvriers et les ouvrières sont de plus en plus nombreux à discipliner : les spécialistes des « engins » sont invités à devenir des cadres.

L'expertise organisationnelle des ingénieurs prend de la consistance avec ce que de nombreux observateurs qualifient à la fin des années 1930 de " révolution managériale " ou "d'ère des organisateurs ${ }^{26}$ ". Les exemples

21. Grelon, 1986, p. 20-21.

22. Cohen, 2013, p. 168.

23. Cheysson, 1897.

24. Dumons et Le Bot, 2010.

25. Lamirand, 1932 ; Dard, 2011.

26. Burnham, 1941 ; Chandler, 1962. 
soviétique et américain suggèrent une bureaucratisation des sociétés modernes, concrétisée par l'émergence d'une nouvelle classe sociale intermédiaire : les " directeurs ${ }^{27}$ ". L'alliage se ferait ainsi entre bureaucratie et technocratie et marquerait la prééminence affectée à l'intelligence des cols blancs sur le savoir-faire des cols-bleus. La grande entreprise apparaît comme le lieu idéal de la mise en application des solutions supérieures, comme le secteur porteur car meilleur, et donc comme un modèle à appliquer par tous. Cela amène les défenseurs de cette nouvelle organisation des entreprises à confondre le dynamisme indéniable de certains secteurs de l'économie avec l'ensemble des forces à l'œuvre dans l'essor fulgurant des nouvelles nations de la seconde industrialisation, au risque d'occulter celles de la première.

Ainsi, c'est au moment où les ingénieurs voient leur titre reconnu qu'ils rencontrent la nouvelle catégorie des cadres (executives), à laquelle ils sont amenés à se mêler. Luc Boltanski a souligné le contraste entre la difficulté à fixer les critères objectifs de l'identité de ce groupe et le fort sentiment d'appartenance de ses membres à une même pratique de management ${ }^{28}$. La Confédération générale des cadres (CGC) est fondée en octobre 1944. Elle représente un groupe en croissance d'environ 500000 membres en $1950^{29}$.

Tous ces changements dans les compétences attendues et requises ne se traduisent pas forcément dans les contenus des formations.

\section{La structuration des systèmes de formation : des chemins de dépendance?}

Dans un vaste panorama historique, Antonio Dias de Figueiredo distingue trois modèles européens d'ingénierie déterminant trois types de statut social de l'ingénieur ${ }^{30}$ : celui des ingénieurs français répondant aux besoins d'une bureaucratie centralisée, bénéficiant d'une formation de haut niveau

27. Rizzi, 1939.

28. Boltanski, 1982, p. 49.

29. Jacquin, 1955 ; Paillat, 1960.

30. Figueiredo et Cunha, 2007 ; Figueiredo, 2014. 
et d'un statut social élevé ${ }^{11}$; le modèle de l'ingénierie civile britannique fondée sur un apprentissage progressif sur le terrain des activités industrielles en développement et organisé autour d'associations sectorielles d'ingénieurs qui en attendent une forme de reconnaissance ; enfin, le modèle de l'ingénierie pratique germanique (puis américaine) qui tente de combiner la recherche et l'enseignement au sein des universités ellesmêmes financées par des institutions externes ${ }^{32}$. La situation des ingénieurs européens s'avère en fait plus diversifiée selon les pays et souvent hybridant ces modèles ${ }^{33}$.

\section{La diversité des systèmes de formation corrélée aux configurations étatiques}

Partout en Europe, l'État joue un rôle décisif dans l'élaboration des systèmes de formation des ingénieurs, enjeu pour les fonctions étatiques régaliennes et le développement national. La variété des situations politiques et de l'organisation administrative des pays européens est l'un des premiers facteurs d'explication de style nationaux d'ingénieurs. L'ingénieur moderne naît au milieu du XVIII ${ }^{\mathrm{e}}$ siècle avec la création et l'essor des premières écoles destinées à former les ingénieurs militaires d'abord puis ceux des grands corps civils d'État, depuis l'École des ponts et chaussées $(1747)^{34}$ jusqu'à l'École des mines (1783). Ces dates correspondent moins à des créations qu'aux rassemblements d'institutions dispersées, à l'image de l'École centrale des travaux publics destinée à fournir les ingénieurs civils et militaires de l'État révolutionnaire : elle est rebaptisée "École polytechnique » en 1795 et recentrée sur les fonctions militaires sous l'Empire. Les contributions dans le dossier d'Alexandre Moatti et d'Hervé Joly soulignent que cette spécialisation n'a pas empêché les ingénieurs de l'X de mettre leurs compétences au service de l'essor industriel. De même, en Espagne, tandis que les deux dernières décennies du $\mathrm{XIX}^{\mathrm{e}}$ siècle furent marquées par une forte

31. Weiss, 1982. Le point de vue de Figueiredo doit être nuancé car il ne considère en fait que les "grandes Écoles", soit une poignée d'établissements. Les Gadzarts qui ont obtenu le diplôme d'ingénieur ("brevet ») en 1907 et qui produisent beaucoup plus d'experts techniques ne disposent pas de ce statut social élevé. Il en est de même pour les ingénieurs issus des instituts techniques universitaires.

32. Locke, 1984.

33. Gouzévitch et al., 2016-2017.

34. Picon, 1992. 
croissance de l'activité entrepreneuriale, pour de nombreux ingénieurs de Caminos (équivalent espagnol des Ponts et Chaussées), le service de l'État fonctionna comme un tremplin pour les postes de dirigeants dans les entreprises municipales et privées en lien avec les travaux publics ${ }^{35}$. Mais l'explosion des nouveaux besoins en ingénieurs civils ne pouvait plus dépendre de quelques détournements de hauts fonctionnaires (pantou(llages $)^{36}$. C'est pour dépasser le monopole d'État qu'est fondée dès 1829 en France, l'École centrale des arts et manufactures de Paris (ECAM).

La formation des ingénieurs français est marquée par cette dichotomie (hiérarchie) entre ceux qui se destinent aux fonctions de l'État et ceux qui optent pour une carrière dans l'industrie. Cette même mutation de l'ingénieur d'État à l'ingénieur civil s'observe partout en Europe occidentale.

L'exemple de la Belgique est sur ce plan significatif. C'est durant la période napoléonienne que sont créés le corps des Ponts et Chaussées et le corps des Mines. À partir de 1835, l'École industrielle de Gand offre l'instruction nécessaire aux arts et manufactures, l'architecture civile et les ponts et chaussées, tandis que l'École des mines de Liège forme aux arts et manufactures et aux mines. Ces deux écoles sont annexées aux facultés des sciences de ces villes les plus industrialisées du pays. L'École royale militaire de Bruxelles forme également des ingénieurs. Parallèlement à ce réseau de formation des ingénieurs d'État, se met en place la formation des ingénieurs destinés à l'industrie dans deux écoles d'arts et manufactures (des mêmes villes de Gand et de Liège), ainsi qu'une École des mines à Mons avec l'appui des propriétaires de charbonnages. Durant la seconde moitié du siècle, d'autres institutions sont créées dans les domaines de l'agriculture, du commerce et de l'industrie. Mais jusqu'en 1888, seuls les ingénieurs diplômés de l'École industrielle de Gand ou de l'École des mines de Liège peuvent exercer au service de l'État. Cette chronologie du déploiement des formations d'ingénieurs se démarque du modèle initial français par les spécificités de l'histoire politique de la Belgique : l'ensemble du système d'enseignement en Belgique est dominé par la dualité linguistique et par l'opposition entre un réseau officiel d'écoles et un réseau catholique ${ }^{37}$.

35. Martykánová, 2009.

36. Charle, 1987.

37. Baudet, 1991, p. 120 ; Brion, 1986, p. 255-256. 
Les formations d'enseignement supérieur portent bien la marque de caractéristiques nationales ${ }^{38}$. Ainsi, l'Italie de la fin du XIX ${ }^{e}$ siècle est confrontée à la question de son existence en tant qu'État unifié et a hérité des anciens États, d'une pléthore d'universités plus ou moins prestigieuses ${ }^{39}$. L'Espagne, quant à elle, est influencée au XviII ${ }^{\mathrm{e}}$ siècle par la France dans la création d'écoles d'ingénieurs militaires et des grands corps civils. Elle ouvre des écoles d'ingénieurs industriels dans tout le pays durant le premier XIX ${ }^{\mathrm{e}}$ siècle (beaucoup dans les années 1850-1855). Celles-ci sont toutefois contraintes de fermer avec la crise économique des années 1865-1867, à l'exception de celle de Barcelone. Le fait que, jusqu'à la fin du siècle, l'unique école industrielle du pays ait été installée, non à Madrid, mais à Barcelone, traduit une division fondamentale au sein du pays entre capitale politique et capitale économique, qui n'a pas été sans effet sur l'industrialisation déséquilibrée de l'Espagne ${ }^{40}$.

De son côté, la stabilité administrative de l'Allemagne à la fin du XIx ${ }^{e}$ siècle et sa structure en Länder se traduisent par la mise en place d'un système d'instituts polytechniques fédéraux ${ }^{41}$. Une dichotomie s'opère dans les pays germaniques entre la voie universitaire (Fachschulen) et une filière des écoles d'ingénieurs (Technischen Hochschulen), elles-mêmes concurrentes. Cette distinction se retrouve en Belgique entre ingénieurs scientifiques et ingénieurs techniciens, en Espagne et en Italie entre ingénieurs et peritos ou peritii. Cela engage une compétition des écoles supérieures techniques en vue d'obtenir la reconnaissance de l'équivalence universitaire et l'établissement d'une science technique autonome.

Paradoxalement, c'est en Angleterre, pays autoproclamé de la « révolution industrielle ", que la formation des ingénieurs semble avoir été la moins sophistiquée. Les industriels britanniques privilégient l'apprentissage et la formation pratique sur le tas, au détriment d'une formation longue dans des établissements spécifiques. Dès 1866, John Fowler, constructeur du premier métro de Londres, se plaint du manque d'instruction des ingénieurs britanniques et proclame la "supériorité » des systèmes d'enseignements français et germanique ${ }^{42}$.

38. Grelon, 1986, p. 20.

39. Lacaita, 1986.

40. Lusa Monforte, 2004 et 2006.

41. König, 2016-2017 ; Stück, 1986.

42. Albu, 1980, p. 67. 
À la fin du XIx ${ }^{e}$ siècle, il n'y avait que quatre universités en Angleterre et au Pays de Galles et quatre autres en Écosse qui offraient un enseignement pour ingénieurs, délivrant peu de diplômes : seulement 1623 en 1908. À la même époque, l'Allemagne comptait environ 30000 ingénieurs formés académiquement et trois à cinq fois plus dans les écoles d'ingénieurs de niveau inférieur, soit un total d'au moins $150000^{43}$. Le contraste de ces chiffres s'avère bien trop important pour traduire un réel différentiel de qualification de part et d'autre de la mer du Nord : la Grande-Bretagne reste alors "l'atelier du monde " et l'apprentissage des ingénieurs y passe tout simplement par d'autres voies que celle du diplôme ${ }^{44}$.

Au début du $\mathrm{xx}^{\mathrm{e}}$ siècle, la référence aux États-Unis s’impose, bien que ce soit la comparaison avec l'Allemagne qui serve, tout particulièrement au moment des deux conflits mondiaux, de motifs à regretter les lacunes du système britannique de formation des ingénieurs. Les mesures prises pendant la Grande Guerre pour rattraper ce « retard » ont été vite abandonnées, faute de moyens. Les rapports du Board of Education incriminent surtout l'absence de demande des industries britanniques en ingénieurs formés. Cette préférence pour une formation sur le tas expliquerait le nombre limité, le faible niveau d'éducation, de rémunération et de considération sociale, des ingénieurs anglais. La grande majorité d'entre eux ne disposait, pour seule qualification que leur appartenance à l'une des associations d'ingénieurs. Aucun examen n'était requis pour y adhérer jusqu'à celui introduit par les ingénieurs civils en 1896 puis pour les ingénieurs de mécanique et d'électricité en 1913. Ce n'est que dans le courant des années 1920 qu'est mis en place un système de certificats : Ordinary National Certificate (ONC) et Higher National Certificate (HNC) qui obtient un certain succès auprès des jeunes étudiants. En 1938, 3313 candidats sur 5797 obtiennent l'ONC et 1137 sur 1668 le HNC. À cette date, quelque 1000 ingénieurs étaient diplômés chaque année au Royaume-Uni. Au début des années 1930, il n'y avait pas plus de 120000 ingénieurs britanniques contre 460000 en Allemagne (dont 100000 diplômés - 3500 ingénieurs sortaient chaque année des Technischen Hochschulen).

Les institutions se connaissent et s'inspirent les unes des autres, mais il n'y a jamais une simple copie - un transfert d'un modèle d'un pays à 
l'autre. Les relations internationales déclinées sous forme de coopérations, de traités, de tensions et de guerres contribuent à l'accélération des échanges. En France la défaite de 1870-1871 conduit à réformer le système de formation technique. En Italie, la Grande Guerre renforce la curiosité pour l'étranger : le ministère de l'Armement fait traduire des ouvrages allemands; des ingénieurs se rendent dans les usines anglaises et américaines ${ }^{45}$. Les visites de mines et d'usines, sous la forme d'un " tour ", contribuent à la formation des ingénieurs ${ }^{46}$. Ces exemples de circulation, à l'instar de ceux pris dans le dossier, permettent à la fois d'insister sur la nécessité d'une approche nationale, voire régionale des dynamiques sociohistoriques, mais invitent également à une montée en généralité pour retenir des tendances communes à une histoire des mondes d'ingénieurs en Europe.

\section{Les trois filières de formation et la hiérarchie des ingénieurs}

Y a-t-il un modèle de " l'ingénieur à la française » caractérisé par une forte compétence théorique et une maîtrise pratique incontestable ? Comme le souligne Éric Godelier dans le dossier, l'École polytechnique a servi d'exemple pour les autres écoles d'ingénieurs en France et en Europe. Pourtant son programme d'enseignement visait à former des cadres supérieurs de l'Armée ou de la Fonction publique. Les compétences visées n'étaient pas celles dont avaient besoin les entreprises industrielles qui ont pourtant employé l'essentiel de ce corps. De fait, il existe plusieurs voies pour former des ingénieurs qu'André Grelon résume en trois filières hiérarchisées ${ }^{47}$. La plus prestigieuse est la filière scientifique des Grandes écoles sur le modèle des corps d'État. Un ingénieur d'État doit être savant et cultivé. Sa formation scientifique valorise l'abstraction par le biais de cours fondamentaux, contrebalancée par une place importante accordée à la culture générale. Comme le suggère Alexandre Moatti, ce mode de sélection se traduit par une forme de condescendance pour les commerçants et les industriels. Les écoles centrales parisienne et lyonnaise dont la mission première était de fournir les cadres de l'industrie, ont eu pour projet de proposer une formation plus pratique en atténuant la distinction entre

45. Dewerpe, 2017, p. 439.

46. Passaqui, 2015.

47. Grelon, 1998. 
sciences et sciences appliquées. Pourtant, rapidement elles reprennent le même mode de recrutement et les mêmes programmes scientifiques que les écoles d'État. Cette formation d'élite pour les élites approfondit la césure avec les autres milieux sociaux et en particulier avec les ingénieurs issus d'une deuxième voie, la filière technique des Arts et métiers.

Cette dernière s'organise d'abord autour de deux institutions : le Conservatoire national des arts et métiers (Cnam, 1794) et le réseau des Écoles des arts et métiers ${ }^{48}$. À la différence des Grandes écoles, les candidats sont issus de l'enseignement primaire supérieur qui s'adresse aux enfants des milieux sociaux moyens ${ }^{49}$. Elle forme d'abord des techniciens qualifiés polyvalents qui assurent l'encadrement intermédiaire dans l'industrie (contremaîtres, agents de maîtrise, etc.). Le CNAM ne délivre des diplômes d'ingénieurs qu'à partir de l'entre-deux-guerres, et encore à l'unité. Le développement des formations d'ingénieurs en tant que telles, y est postérieur à la Seconde Guerre mondiale. Par ailleurs, en 1816, est créée à Saint-Étienne une école pour former des maîtres-mineurs, autres techniciens intermédiaires. Il faut attendre 1891 pour que l'École des mines puisse délivrer un titre d'ingénieur. Celle-ci forme des "ingénieurs expérimentateurs " porteurs d'une culture scientifique industrielle, placés à la charnière du public et du privé et qui restent des agents secondaires du Corps des mines ${ }^{50}$. Le réseau des écoles nationales professionnelles (ENP) se déploie dans le pays à partir de 1881 pour être le vivier de l'aristocratie technicienne et la voie royale d'accès aux Arts et métiers ${ }^{51}$. Durant les années 1930, ces derniers ne délivrent qu'un «brevet » d'ingénieur (voir Alain P. Michel à propos de Pierre Bézier), le "diplôme » n’apparaissant qu'après-guerre. L'ensemble de ces organismes techniques offre la possibilité pour un technicien supérieur de se métamorphoser en ingénieur, quoique de catégorie "inférieure ».

Enfin, une troisième filière spécialisée se met en place à la fin du $\mathrm{XIX}^{\mathrm{e}}$ et au $\mathrm{xx}^{\mathrm{e}}$ siècle. À partir des années 1880 , un grand nombre d'écoles d'ingénieurs (notamment en physique-chimie et en électricité) sont créées du fait d'une demande croissante et inédite : quarante-trois entre 1880 et 1918. C'est le cas de l'École de physique et de chimie industrielles

48. Day, 1991.

49. Grelon, 1998, p. 249.

50. Garçon, 2004.

51. Brucy et Le Bot, 2015. 
(EPCI) fondée en 1882 par la ville de Paris, considérée comme la première école d'ingénieurs " moderne " française (même si elle n'en a pas immédiatement le statut), prototype des établissements d'impulsion municipale qui prennent en compte un nouveau type de demandes industrielles de la seconde industrialisation ${ }^{52}$. Cette filière se développe entre 1919 et 1936 avec vingt-trois autres fondations, marquant l'essor des écoles d'ingénieurs de province, comme celles de Lorraine ou de Grenoble, dont le rôle a été déterminant dans le panorama national ${ }^{53}$. Les besoins nouveaux incitent les pouvoirs publics nationaux et locaux à encourager ces créations, avec l'appui des organisations patronales. En outre, comme le souligne Virginie Fonteneau dans le dossier, dès l'entre-deux-guerres, les universités entrent progressivement dans la course de la formation des ingénieurs, grâce à leur monopole sur les doctorats. À quelques différences près, le système d'organisation de la filière scientifique est décliné au niveau de tous ces établissements qui cherchent, ce faisant, la reconnaissance.

Cette prégnance du modèle des grandes écoles est une spécificité française. Mais avec un système différent, on retrouve ailleurs en Europe les mêmes distinctions entre filières techniques, scientifiques et spécialisées. En Allemagne, ce sont dès le départ les universités qui accaparent la formation scientifique.

Les pays européens qui ont copié le modèle français ne l'ont toutefois pas maintenu. En Belgique, la loi sur la collation des grades académiques de 1890 crée en particulier les grades d'ingénieur civil des mines et d'ingénieur des constructions civiles. Certaines écoles se trouvent désormais intégrées aux universités qui jusqu'à cette date ne formaient leurs étudiants qu'aux carrières de l'enseignement et aux professions libérales. En outre, la législation ouvre désormais l'accès des ingénieurs civils à la fonction publique, faisant perdre leur monopole aux écoles de Gand et de Liège. La législation de 1890 organise pour près d'un siècle le haut enseignement technique en Belgique. Elle introduit un clivage entre ingénieur issu d'une université, se réclamant de la science, et ingénieur formé hors université, cantonné au champ de la technique ${ }^{54}$. En Espagne, pendant tout le pre-

52. Shinn, 1981 ; Grelon, 1998, p. 236 et 245 ; Emptoz, 2010. L'ECPI devient en 1948 École supérieure de physique et de chimie industrielles (ESCPI) de la ville de Paris, puis ESPCI Paris Tech en 2008 et ESPCI Paris en 2016.

53. Grelon et Birck, 1998 et 2006 ; Robert et Moret, 2001 ; Birck, 2016-2017.

54. Baudet, 1991, p. 120 ; Brion, 1986, p. 255-256. 
mier $\mathrm{Xx}^{\mathrm{e}}$ siècle, les écoles de Barcelone, Bilbao (1899) et Madrid (1902) sont les seuls lieux de formation à la profession d'ingénieur industriel. Il faut attendre les années 1960, pour que de nouvelles écoles apparaissent, en lien avec la nouvelle phase d'industrialisation du pays.

À la suite de la Seconde Guerre mondiale s'observent partout une multiplication des écoles et un élargissement des voies d'accès au statut d'ingénieurs en vue de fournir les cadres nécessaires à une activité industrielle en expansion. À la Régie Renault, le tournant de la production de masse de l'après-guerre s'accompagne du gonflement des effectifs des services existants et suscite l'émergence de services nouveaux ${ }^{55}$. En 1939, on recense en France 10000 ingénieurs, tandis qu'en l'an 2000 il y en a 700000 : l'essor numérique s'est accompagné d'une fragmentation du groupe social ${ }^{56}$.

\section{Les groupes d'ingénieurs et leur socialisation}

\section{Les associations d'ingénieurs en Europe}

Les systèmes d'identification des ingénieurs sont extrêmement divers en Europe. Au pragmatisme britannique qui veut que ce soient les associations d'ingénieurs elles-mêmes qui sélectionnent leurs membres et leur attribuent le nom d'ingénieur après le passage par une série de filtres (parrainages, examens), s'oppose le mode tout à fait formalisé de formation des ingénieurs allemands.

Dès 1818, la fondation de l'Institution of Civil Engineers (ICE) ${ }^{57}$ accompagne l'émergence en Angleterre de la catégorie des ingénieurs au service des entreprises privées. Elle poursuit dans une certaine mesure une première Société des ingénieurs civils fondée dès 1771 sous la forme d'un club de gentlemen. L'ICE, ainsi que la Société des ingénieurs des constructions mécaniques (1847) et électromécaniques (1871), constituent les trois principales associations d'ingénieurs parmi une cinquantaine installée en Angleterre avant $1930^{58}$. Dans une société victorienne où la respectabilité

55. Fridenson, 1985 et 2007.

56. Derouet et Paye, 2018.

57. Ferguson et Chrimes, 2011.

58. Marsh, 1986, p. 241-242. 
importe, ces associations jouent un rôle de parrainage pour leurs adhérents. John W. C. Haldane précise ainsi en 1887 :

On trouve chez les jeunes gens des institutions d'ingénieurs, toutes les extractions, tous les degrés de sens moral et compétence (du plus élevé au plus insignifiant). L’admission est obtenue par trois moyens : premièrement par relations, deuxièmement par l'argent, troisièmement par la combinaison des deux ${ }^{59}$.

La dimension de qualification importe cependant à ces associations et la qualité de membre de plein droit était réservée aux " experts " possédant une expérience d'au moins cinq ans d'exercice. En revanche, les associations anglaises d'ingénieurs ne furent pas en mesure de contrôler la formation. L'idée d'un registre des ingénieurs britanniques qui aurait permis de fermer l'accès de la profession, est écartée à la fin du XIX ${ }^{e}$ siècle. À cette époque, les associations d'ingénieurs utilisèrent, à l'instar d'autres secteurs, l'examen d'entrée afin de déterminer pour leurs stagiaires un niveau de qualification. C'était aussi une manière de certifier a posteriori l'enseignement reçu dans les écoles techniques. Mais le contrôle ne fut jamais total, toujours modéré par l'influence des pouvoirs publics : la possibilité pour les étudiants de devenir membres des différentes sociétés d'ingénieurs restait secondaire par rapport à l'acquisition de compétences ${ }^{60}$. Il faut attendre les années 1920 pour que les associations d'ingénieurs se résolvent à protéger leur titre en usant de l'expression Chartered Engineer - ingénieur agréé ${ }^{61}$. L'échec des associations en matière de contrôle de la profession, s'explique sans doute par une préoccupation privilégiée pour le progrès et la diffusion des connaissances scientifiques et techniques. En s'organisant autour de la production d'un savoir, ces collectifs manquèrent les opportunités de contrôler leur discipline et d'élever le statut de leurs membres. En outre, ils durent finalement renoncer, pour l'essentiel, à la production de connaissances au profit d'universités qu'ils avaient pourtant négligées ${ }^{62}$. Ainsi, au pays du libéralisme, les ingénieurs commencent à s'organiser afin de s'arroger le monopole de la désignation des ingénieurs. Mais leurs institutions perdent peu à peu le contrôle de la profession, que ce soit au niveau de l'enseignement supérieur ou pour le recrutement par les industriels.

59. Haldane, 1887.

60. Marsh, 1986, p. 247-248.

61. Ibid., p. 247.

62. Ibid., p. 252. 
En Belgique, la première association d'ingénieurs civils remonte à $1847^{63}$. Dans la foulée, la Société centrale des ingénieurs civils (SCIC) est fondée en France le 4 mars 1848, à l'initiative d'un groupe d'anciens élèves de l'ECAM. Elle devient en 1850 la Société des ingénieurs civils de France. La disparition de la référence à " centrale " marque le souci de ne pas limiter l'association aux seuls élèves de l'ECAM : ingénieur civil s'oppose ici aux ingénieurs de la fonction publique formés au sein de Polytechnique et de ses écoles d'applications, civiles ou militaires. À côté des buts d'information et d'entraide, la SCIC se donne comme objectif :

C 6 de faire connaitre et apprécier le corps des ingénieurs civils, en signalant les services déjà rendus par les membres de ce corps, ceux, plus importants encore, qu'ils pourront rendre; de poursuivre la juste satisfaction de ses intérêts et de ses droits, fondés sur des garanties sérieuses de capacité ; de réclamer une large part dans les fonctions publiques et les travaux de l'État ; etc. ${ }^{64}$

Dans le même esprit est créée aux États-Unis en 1852, l'American Society of Civil Engineers (ASCE) et en Allemagne en 1856, la Verein Deutscher Ingenieure (VDI).

Au fur et à mesure de leur croissance, les associations d'ingénieurs européennes installèrent des comités de spécialistes pour étudier des problèmes techniques spécifiques. Cela s'inscrivait dans la tendance générale d'établissement des normes techniques de la pratique de l'ingénieur (voir dans le dossier, l'Union électrotechnique tchécoslovaque étudiée par Marcela Efmertova). En Angleterre, les ingénieurs civils, mécaniciens et autres, créèrent en 1901 le Comité des Normes de l'Ingénieur, devenu en 1918, l'Association Britannique des Normes de l'Ingénieur, avant de devenir en 1931 le British Standards Institution ${ }^{65}$. À travers leur activité de standardisation, ces associations orientaient leur profession vers l'organisation et la protection des sciences de l'ingénieur ${ }^{66}$.

63. Baudet, 1991, p. 124.

64. Société centrale des ingénieurs civils, 1848.

65. Dubreuil, 1929.

66. Marsh, 1986, p. 246. 


\section{La constitution de réseaux de sociabilités distincts : ingénieurs civils vs industriels}

Les associations d'ingénieurs caractérisent un besoin de s'unir pour défendre des intérêts spécifiques. C'est le cas par exemple de la fondation en France dès 1846 de la Société des ingénieurs arts et métiers, sans doute la première association française d'anciens élèves. Cette précocité s'explique à la fois par l'histoire déjà ancienne de cette école et par le besoin de ses membres de défendre la légitimité de leur titre. Ce réseau social a toujours été un soutien pour la carrière de ses élèves. Son efficacité n'est pas étrangère à l'accès de l'ENSAM au rang de grande école. C'est aujourd'hui en Europe, l'association qui fédère le plus d'adhérents pour un même établissement d'enseignement supérieur, avec quelque 34000 membres !

Les associations d'écoles sont pour la plupart corporatistes. C'est le cas, par exemple, de la Fédération des Associations Belges d'Ingénieurs (FABI) fondée en 1926 par les associations des six grandes écoles de niveau universitaire et dont le premier objectif est la protection du titre d'ingénieur ${ }^{67}$. Depuis le $\mathrm{XIX}^{\mathrm{e}}$ siècle, il existe en Belgique deux formes d'associations, les unes rattachées aux universités et les autres aux écoles d'ingénieurs techniciens. Les premières sont élitistes et s'attachent aux manifestations extérieures de prestige : rentrées académiques solennelles, port de la toge par le corps professoral, publications nombreuses et prestigieuses, etc. Elles ont pour but la diffusion des idées ; elles sont très soucieuses de leur prestige et plutôt fermées. Le modèle en est la Société Royale Belge des Ingénieurs et Industriels (SRBII) fondée en 1885 à l'exemple de la Société des Ingénieurs Civils de France. Elle rassemble des personnalités sur la base de leurs réalisations industrielles ou scientifiques et en ne tenant pas compte de leur formation initiale. Pour les secondes, plus corporatistes, l'enjeu n'est pas "l'invention d'une tradition ${ }^{68}$ ": la pompe est quasi inexistante (peu de séances publiques par exemple) et la recherche et les publications sont bien moindres. Elles ont pour but statutaire la défense des intérêts de leurs membres et sont principalement soucieuses d'attirer le plus d'adhérents possible. Elles recrutent parmi les écoles techniques qui sont apparues au tournant des $\mathrm{XIX}^{\mathrm{e}}-\mathrm{XX}^{\mathrm{e}}$ siècles sur le modèle des écoles françaises d'arts et

67. Baudet, 1991, p. 124-125; Brion, 1986, p. 258.

68. Hobsbawm, 2006. 
métiers à l'initiative des industriels : l'École des textiles de Verviers (1892), l'École centrale des arts et métiers (Bruxelles, 1905), l'Institut Gramme (Liège, 1906) ${ }^{69}$.

L'accroissement de la production à la suite de la Première Guerre mondiale accéléra la concentration industrielle, la rationalisation des entreprises, les multiplications des degrés hiérarchiques et pour y pourvoir, un développement important de ces formations hors universitét ${ }^{70}$. Cela n'est pas sans conséquence sur l'origine socio-économique des étudiants fonction de la nature des écoles et accentue l'opposition entre ingénieurs civils et ingénieurs industriels. Il en découle que les uns et les autres se distinguent assez facilement, au moins en début de carrière, par des traits de comportement qui définissent deux sous-cultures, et qu'un conflit permanent s'installe qui porte évidemment sur l'accès aux carrières les plus brillantes et aux postes les plus rémunérateurs de l'industrie et de l'administration ${ }^{71}$.

\section{La féminisation des mondes d'ingénieurs au $\mathrm{XX}^{\mathrm{e}}$ siècle?}

En 1972, Anne Chopinet est reçue major à Polytechnique, la première année d'admission des femmes: "Vous n'avez pas peur qu'on vous prenne pour un monstre et qu'on s'éloigne de vous ? " lui demande le reporter du journal télévisé du 2 août $1972^{72}$...

Les femmes sont longtemps rares, mais elles ne sont pas absentes parmi les ingénieurs. Ainsi, entre 1893 et 1939, 186 élèves sont sortis diplômés de l'École de céramique de Sèvres, mais seulement cinq femmes ont suivi le cursus et trois ont reçu le titre ${ }^{73}$. Aux Mines de Saint-Étienne comme à Polytechnique, rappelle Hervé Joly, les femmes sont exclues par le règlement jusqu’au début des années 1970. De toute manière, seuls les lycées de garçons disposent de classes préparatoires scientifiques. À l'étranger, les chiffres des années 1970 montrent une situation comparable dans les pays à économie libérale, tandis que le pourcentage de femmes ingénieures dans

69. Brion, 1986, p. 260.

70. Ibid.

71. Baudet, 1991, p. 122.

72. "1972 : Une femme reçue à l'école Polytechnique ? ", Archive INA, consultée le 15 mars 2020, https://www.youtube.com/watch?v=zTIWpWyS6sc.

73. Le Bot, 2016. 
les pays communistes du bloc de l'Est semble plus important : ainsi, en Pologne, $30 \%$ des ingénieurs du textile et de la chimie sont des femmes ${ }^{74}$.

L'entrée des femmes dans les écoles d'ingénieurs relève, selon Catherine Marry, d'une "révolution respectueuse " en trois étapes ${ }^{75}$. La première est celle des pionnières, de 1920 aux années 1960. Les écoles d'ingénieurs fondées à la suite de la Première Guerre mondiale ont toutes été " mixtes » (École de chimie de Marseille, 1917 ; Rouen, 1918 ; Rennes, 1919 ; Strasbourg, 1920 ; Institut d'optique, 1920) ; quelques écoles, fondées antérieurement, se mettent également à accepter les filles (ECAM, 1917 ; Institut national d'agronomie, École supérieure d'électricité, 1919 ; EPCI, 1922 ; École supérieure d'aéronautique, 1924$)^{76}$. On ne peut cependant pas parler d'une véritable mixité, car le mouvement était très faible et les cas de filles admises étaient souvent uniques (seules une cinquantaine de femmes obtiennent le diplôme de Centrale Paris pendant l'entre-deuxguerres). En 1925, Marie-Louise Paris (ingénieure diplômée de l'École de mécanique et d'électricité de Paris, ainsi que de l'Institut électronique de Grenoble), fonde une école d'ingénieures réservée aux femmes : l'Institut électromécanique féminin, qui devient en 1933, l'École polytechnique féminine (Sceaux). Mais là encore, les effectifs restent faibles : cinq diplômées par an de 1927 à 1939 ; un peu plus de six de 1940 à 1944 ; près de 21 de 1945 à $1949^{77}$. Les autres écoles, plus anciennes et réputées, ferment les portes aux femmes. Leur proportion parmi les ingénieurs est de l'ordre de $3,5 \%$ en $1963^{78}$.

La deuxième étape, telle que définie par Catherine Marry, est celle plus courte des générations de transition des années 1970, où les écoles d'ingénieurs s'ouvrent aux candidates à l'image d'Anne Chopinet. Enfin, la troisième est celle d'une banalisation inaboutie, en matière salariale d'abord et du fait de la difficulté des femmes à accéder aux sphères supérieures des entreprises (le "plafond de verre »). C'est dans ce contexte que l'association française des femmes ingénieurs est créée en 1982, devenue en 2009,

74. Peslouan, 1974.

75. Marry, 2004.

76. Stevanovic, 2004.

77. Grelon, 1992.

78. Peslouan, 1974. Concernant l'accès aux diplômes et aux titres, la problématique des pionnières ou la mesure de la participation des femmes aux professions scientifiques à l'université et dans la recherche privée, Rossiter, 1982 et Gardey, 2000. 
Femmes ingénieurs (FI). Son objet est la promotion de la place des femmes ingénieures et scientifiques dans le monde du travail ; la promotion du métier d'ingénieur auprès des jeunes, filles et garçons, dans le monde de l'éducation ; la représentation des femmes exerçant les métiers de l'ingénieur dans la société. En 2010, La part des femmes ingénieures en France est d'environ $17 \%{ }^{79}$.

Sur ces questions, les sciences sociales (notamment Delphine Gardey et ses collègues) ont étoffé leur questionnaire pour interroger les sciences et les techniques dans le rôle joué en matière d'exclusion, à travers leurs institutions, leurs lieux et leurs modalités d'exercice, leurs professionnalisations, leurs représentations ${ }^{80}$. L'absence des femmes dans les mondes scientifique et technique est notamment abordée par la mise en évidence de traits structurels des sociétés occidentales - domination masculine, exclusion durable des filles de l'accès à la formation, construction de l'identité sociale des femmes bourgeoises au sein de la seule sphère privée ; par l'étude de mécanismes sociaux plus subtils qui contribuent, par exemple, à l'intériorisation par les filles et les femmes scientifiques des rôles sociaux dominants ; par l'analyse des mécanismes institutionnels discriminants ; par l'analyse des lieux de science et de technique en tant que mondes masculins ${ }^{81}$; par la spécification de l'entreprise scientifique moderne comme une entreprise masculine d'emprise et de domination de la nature - et ainsi des femmes qui furent historiquement assimilées et assignées à la nature et exclues de la raison ${ }^{82}$.

\section{Les ingénieurs en action}

\section{Les ingénieurs : combien et quelles positions dans l'entreprise?}

Longtemps, l'ingénieur est un homme (plutôt qu'une femme) rare. Il occupe une position particulière dans le travail et l'organisation de la production, ce qui explique la faiblesse de sa présence numérique dans

79. Lumineau, 2011.

80. Canel et Zachmann, 1999 ; Chabaud-Rycher et Gardey, 2002 ; Gardey, 2005.

81. Berner, 1997 ; Gardey, 2005.

82. Gardey et Löwy, 2002 ; Gardey, 2005. 
l'entreprise. Dans l'Ansaldo (sidérurgie, métallurgie, mécanique, chantiers navals), groupe industriel installé à l'ouest de Gênes et étudié par Alain Dewerpe, on dénombre en 1916, 95 ingénieurs dans l'ensemble des établissements pour un peu moins de 23000 ouvriers (sans compter les employés), soit environ un ingénieur pour 240 ouvriers $^{83}$.

Soulignons, que bien que peu reconnus et difficilement identifiables, les ingénieurs autodidactes ont joué partout en Europe, un rôle essentiel dans le fonctionnement efficace de leur entreprise. Les cloisons sont poreuses entre l'ingénieur et le contremaître ${ }^{84}$. Chez Renault comme chez Peugeot, tout ingénieur commence sa carrière par un stage ouvrier - une pratique qui perdure dans de nombreux secteurs industriels ${ }^{85}$.

De multiples études historiques sur les ingénieurs des différents domaines sectoriels éclairent la variété de leurs activités selon qu'ils interviennent dans la chimie, les chemins de fer, les industries électriques, les télécommunications, etc. ${ }^{86}$. L'exhaustivité en la matière n'est pas possible, tant prolifèrent les monographies d'entreprises, les études régionales, ou même les biographies d'ingénieurs, pour la France et pour l'Europe de l'ouest (pour nous en tenir aux limites fixées par la question aux concours) ${ }^{87}$.

Par ailleurs, l'historiographie peine encore à mesurer le flux des ingénieurs vers les PME (et plutôt les moyennes entreprises) qui constituent l'essentiel du tissu économique français et même européen de manière durable encore $\mathrm{au} \mathrm{xx}^{\mathrm{e}}$ siècle. Trouve-t-on des ingénieurs dans ces très nombreux établissements ? Les créations d'écoles d'ingénieurs dans certains secteurs dominés par des PME (en 1893, École de céramique de Sèvres ${ }^{88}$ et École de brasserie et de malterie à Nancy, ou encore École de tannerie de Lyon en 1899) sont des indices en la matière. Il faudrait ensuite regarder de plus près comment les emplois se répartissent d'un point de vue sectoriel, mais

83. Dewerpe, 2017, p. 435.

84. Schweitzer-Vandecasteele, 1990.

85. Cohen, 2001 ; Michel, 2021.

86. Sans viser aucunement à l'exhaustivité, pour la chimie : Lamard et Stoskopf, 2010 ; Emptoz et al., 2018. Pour les chemins de fer, notamment Ribeill, 1993. Pour l'électricité, Bouneau, 1995. Pour les télécommunications, Griset, 2013.

87. Parmi bien d'autres, Daviet, 1988 ; Dhombres, 1994 ; Angio (d'), 2000 ; Bouvier, 2016. Pour des exemples de monographies d'entreprises étrangères : Lewchuk, 1987 ; Collins et Stratton, 1993 ; Church, 1994 ; Mommsen et Grieger, 1996 ; Bigazzi, 2000 ; Kuhr-Korolev and Schlinkert, 2009. 88. Le Bot, 2016. 
également s'agissant de la nature des fonctions occupées (chef de fabrication, directeur d'usine, etc.). Les dynamiques d'innovation sectorielles trouveraient à s'éclairer en regard de l'apport de ces ingénieurs : ainsi par exemple de la fabrique de chaussures de sécurité Jallatte, installée dans les Cévennes, et qui, pour résister à la concurrence internationale, s'est associée à partir de 1960, les services d'ingénieurs aux spécialités variées (chimie, électricité, informatique, mécanique) ; elle est aujourd'hui l'une des principales firmes mondiales sur son marché ${ }^{89}$.

Une approche territoriale s'impose également quand les sciences sociales ont désormais bien repéré les logiques spatiales et même les logiques de proximité à l'œuvre dans bien des secteurs économiques. À la fin du $\mathrm{XIX}^{\mathrm{e}}$ siècle, la demande en techniciens et ingénieurs qualifiés en électricité se fait pressante dans la région de Grenoble. Fortement dépendantes des particularités régionales que sont les demandes en mécanique et en hydraulique, les écoles grenobloises d'ingénieurs dispensent des enseignements résolument tournés vers la satisfaction de l'industrie locale, avant de répondre aux demandes de l'ensemble de l'industrie électrotechnique française ${ }^{90}$.

La position des ingénieurs n'est pas acquise au sein de l'entreprise. Le diplôme ne garantit pas la fonction : titre et emploi sont dissociés. Ces derniers sont fortement contrastés entre ingénieur de conception (dans les bureaux d'études, au siège des firmes ou à la tête d'établissement) et ingénieur d'atelier, de production. Dans l'Ansaldo italien, les postes de direction ne sont pas forcément occupés par des ingénieurs, mais peuvent l'être par des cadres d'ateliers non diplômés. Ceci correspond à une méfiance pérenne à l'égard des apports de la science, des capacités de l'école et de la valeur du diplôme ${ }^{91}$. De même, jusqu'aux années 1930, rares sont les cas où les services d'un ancien élève de l'École nationale supérieure de céramique aient été employés par les fabricants de Limoges, capitale française de la porcelaine, même si ces patrons d'entreprises, parfois importantes, sont demandeurs de connaissances scientifiques et techniques pour améliorer leur procès de production et leurs produits ${ }^{92}$. Connaissances théoriques et savoirs pratiques sont ainsi en tension ; l'aptitude à exercer, reconnue par

89. Le Bot, 2021.

90. Robert et Moret, 2001.

91. Dewerpe, 2017, p. 437.

92. Le Bot, 2016. 
un diplôme, n'équivaut pas pour les recruteurs à la capacité à pratiquer selon les règles de l'art : il y faut selon eux l'expérience de la fabrique.

Les ingénieurs les plus entreprenants créent leurs entreprises sur place ou bien à l'international. Ainsi, durant le premier $\mathrm{XIX}^{\mathrm{e}}$ siècle, s'installent en Italie des ingénieurs appelés à y fonder des entreprises importantes : les français Joseph-Marie et Jean Balleydier qui ont créé le premier complexe industriel à Gênes en 1832 ; deux autres frères, James et Joseph Westermann, originaires de Manchester, qui ont ouvert des chantiers navals dans la même ville en 1846 ; l'ingénieur écossais Thomas Robertson, qui établit à la même époque une autre grande usine de mécanique, toujours à Gênes ; ou encore Thomas Richard Guppy, un ingénieur et industriel de Bristol qui s'implante à Naples en $1849^{93}$. Ce rôle dans la diffusion industrielle est l'objet de plusieurs contributions de ce dossier. Bertrand Eychenne montre par exemple comment le développement minier de la Colombie est apparu comme un eldorado pour un ingénieur-savant français qui s'est mis au service d'une compagnie d'investissement anglaise et pour un ingénieur-expert qui y a fondé une compagnie minière française.

L'ingénieur est un passeur : concepteur d'améliorations quand il en a l'opportunité, introducteur d'innovations en entreprise, vecteur de changements techniques dans les sociétés, diffuseur de configurations industrielles à travers les frontières des États.

\section{Penser par cas : de Brunel à Bézier}

La meilleure formation et la solution technique la plus élégante ne sont pas toujours celle qui s'impose. C'est la mésaventure qui arrive à un ingénieur X-Mines qui entre 1822 et 1832 , installe et perfectionne deux machines à colonnes à eau particulièrement sophistiquées. Le dispositif permet de pomper l'eau de la mine d'Huelgoët (Bretagne) sans houille ni feu. Cette " machine à vapeur sans vapeur " est un exploit technique qui matérialise l'aptitude de l'ingénieur d'État à surmonter les contraintes imposées par le site. Mais «l'engin naquit bloqué parce que trop en adéquation avec un milieu et insuffisamment adaptable [...]. L'industrie dédaigna cette haute couture mécanique. Ce que recherchaient les entrepreneurs était bien plutôt un prêt-à-porter bon marché, des machines sans histoire 
qu'ils trouvèrent, dans le secteur minier, sur le marché de l'occasion des machines à vapeur britanniques ${ }^{94} »$. Toutes les compétences d'ingénierie ne sont pas adaptées à tous les chantiers dans lesquels les ingénieurs agissent. Ils doivent s'adapter aux spécificités de chaque secteur industriel. La grande compétence scientifique de l'ingénieur d'État doit être en phase avec le monde de la production. Aux ingénieurs d'État l'administration de la science industrielle. Aux ingénieurs de l'École centrale, à ceux des filières techniques, aux savants des laboratoires, voire aux autodidactes, le soin d'améliorer et plus rarement d'innover. Dans cette perspective, il n'est pas dit que la poussée inventive du $\mathrm{XIX}^{\mathrm{e}}$ siècle ait connu deux époques et deux styles chronologiquement séparés, le temps des découvertes des artisans praticiens anglais de la première industrialisation et celui des ingénieurs et des savants de la seconde. Les " retards » relèvent peut-être plutôt de systèmes techniques distincts.

L'ingénieur est une figure de la modernité, quelque part entre le « capitaine d'industrie » et le savant ${ }^{95}$. Il mérite que l'on relève, au même titre que pour d'autres figures, le "pari biographique ${ }^{96}$ ", ne serait-ce que pour éclairer des pans importants de l'histoire économique, industrielle, scientifique et technique qui risqueraient sinon de demeurer dans l'ombre.

Ainsi, l'ingénieur Isambard K. Brunel (1806-1859) est une des figures emblématiques de l'Angleterre victorienne, qui a porté la foi dans les sciences et les techniques à son acmép7. Brunel est d'abord connu pour avoir créé le Great Western Railway (ligne de chemin de fer LondresBristol). Les projets de Brunel (ponts, tunnels, navires transatlantiques, etc.) ont souvent été accompagnés de solutions novatrices, apportant des réponses à long terme à bien des problèmes d'ingénierie. Sa réalisation la plus connue est le pont suspendu de Clifton à Bristol : d'une longueur de plus de $213 \mathrm{~m}$, et surplombant de $61 \mathrm{~m}$ la rivière Avon, il détenait à son inauguration le record de la plus longue portée au monde. Son navire à vapeur SS Great Eastern a été le premier à immerger avec succès un câble télégraphique à travers l'océan Atlantique. En 2006, un important programme de manifestations au Royaume-Uni a célébré le bicentenaire de sa naissance. L'ingénieur est honoré par de très nombreux monuments dans

94. Garçon, 2012, p. 177 et 174.

95. Avec tous les biais que cela implique : Le Bot, 2015.

96. Dosse, 2005.

97. Vaughan, 2006. 
tout le pays et l'acteur Kenneth Brannagh l'a même "incarné » lors de la cérémonie d'ouverture des Jeux olympiques de 2012 à Londres, en évoquant la " révolution industrielle " britannique. Cela étant signalé pour mettre en exergue l'importance du personnage outre-Manche, sans rentrer dans le détail des limites et des biais de cette " héroïsation $"^{98}$.

Pierre Bézier (1910-1998) est quant à lui un "inconnu célèbre ». Alain P. Michel propose de confronter ses mémoires personnelles avec les traces laissées dans les archives de l'entreprise Renault afin de cerner la portée de ses actions concrètes ${ }^{99}$. Cette reconstitution du passé de Bézier en fait un " cas " au sens défini par Jean-Claude Passeron et Jacques Revel. La casuistique est une forme concrète de pensée, communément utilisée pour traiter les affaires morales, religieuses ou juridiques. Face aux problèmes posés par une action concrète, la pensée par cas vise à les résoudre grâce à une discussion entre deux types d'éléments : d'une part, les particularités du cas réel étudié, et d'autre part, des principes généraux ou des cas similaires. Ce faisant, la singularité d'une situation est confrontée au suivi temporel de l'histoire dont elle est le produit ${ }^{100}$. Des phénomènes qui ne sont pas directement observables à partir de telle ou telle source, peuvent, par une reconfiguration théorique des concepts, devenir concevables et par là, être rendus descriptibles ${ }^{101}$. Il devient alors possible de faire sortir les ingénieurs de l'ombre de l'action collective à laquelle ils participent de façon anonyme et dont la manifestation publique est le plus souvent (et commodément) attribuée au seul patron " héroïque ». Salariés de la recherche, ils sont au cour du travail d'invention (découverte de nouveaux procédés) et d'innovation (leur application effective) permettant à l'entreprise de renouveler son offre. Praticiens des améliorations et garants de la qualité, ils sont aux fondements de l'adaptation des entreprises et de leur capacité à survivre. Partenaires de la direction à différents échelons, le témoignage d'Yves Dubreil dans le dossier souligne combien les ingénieurs en partagent le pouvoir et, par les "perturbations » qu'ils apportent aux routines, contribuent au dynamisme créatif. Le portrait d'un ingénieur ne se contente pas d'ajouter une nuance à la galerie des figures et de fonctions

98. MacLeod, 2007.

99. Michel, 2015 et 2021.

100. Passeron et Revel, 2005, p. 17-18.

101. Ibid., p. 44. 
connues. Les spécificités dont il témoigne invitent à repenser notre perception historienne des terrains sur lesquels il travaille.

L'analyse que nous élaborons dans le dossier part de l'hypothèse micro-historique qu'au ras des bureaux et des ateliers parcourus par l'ingénieur se nouent les intrigues d'un système de production en mutation. Nous confrontons les schémas de la pensée industrielle d'une époque (scientisme et technicisme, taylorisme, fayolisme, etc.) aux pratiques réelles du terrain usinier. Nous mettons en perspective des actions localisées, dans le contexte changeant de leur concrétisation. Nous opérons un va-et-vient du cliché individuel à la fresque sociale par un processus croisé de montée en généralité et de descente en singularité. Cette navette vise à répondre au problème classique des rapports entre le particulier et le général, en vérifiant l'ancrage dans le réel de la généralisation élaborée à partir de l'observation des faits documentables du passé. La biographie des ingénieurs offre les moyens d'expérimenter cet entre-deux.

L'Europe occidentale voit l'émergence du groupe des ingénieurs autour de deux types de besoins communs : la nécessité de disposer d'un corps technique de l'État en expansion, d'une part et celle de répondre à l'essor industriel, d'autre part. Ce groupe social partage une même culture technique qui combine la maîtrise de théories abstraites et de pratiques concrètes, un fond de sciences exactes et un souci de leurs applications. Les ingénieurs européens ont des rapports complexes aux humanités et ont le même désir d'instituer une " République des Ingénieurs ${ }^{102}$ ", héritée des utopies technicistes du XIX ${ }^{\mathrm{e}}$ siècle, partout cruellement déçu ${ }^{103}$.

Le dossier souligne qu'au-delà des modèles nationaux et des filières de formations, les ingénieurs contemporains combinent quatre dimensions des pratiques d'ingénierie ${ }^{104}$. Autour des engins de production, ils restent des praticiens des arts et métiers de l'artisanat et de l'industrie. Par leurs capacités ingénieuses, ils conçoivent des améliorations pratiques, mais peuvent également trouver le chemin de l'innovation. Le génie industriel, appuyé sur les sciences fondamentales, leur permet de penser le processus de fabrication, d'en analyser les mécanismes, d'en anticiper le fonctionnement et éventuellement d'en réorienter le cours. Enfin, l’ingénieur contemporain

102. Marnot, 2000.

103. Grelon, 1986, p. 239.

104. Figueiredo, 2014. 
découvre le management et, avec l'appui des sciences sociales et humaines, mobilise son rôle de cadre.

Durant le $\mathrm{xx}^{\mathrm{e}}$ et encore au début $\mathrm{du} \mathrm{XxI}^{\mathrm{e}}$ siècle, les grandes entreprises industrielles connaissent de profondes transformations et deviennent des organismes qu'une seule personne ne peut saisir dans sa totalité. Nicolas Hatzfeld dans ce dossier, insiste sur l'usine comme espace mouvant, où se croisent des logiques individuelles et collectives, où se combinent intérêts personnels et des groupes de pression, où se reconfigurent des impératifs techniques et économiques. Les limites de l'usine sont floues et ses éléments constitutifs en perpétuelle reconfiguration. La figure de l'ingénieur, la catégorie d'ingénieur, sont sans doute également à analyser selon ce prisme.

\section{Sources}

Bureau international du travail, Les conditions de vie des ingénieurs et des chimistes, Genève, 1924.

Burnham James, The Managerial Revolution: What is Happening in the World, John Day Company, New York, 1941.

Cheysson Émile, "Le Rôle social de l'ingénieur, réception par la Société des ingénieurs civils de France de la Société d'économie sociale ", le 20 mai 1897, réédité dans Euvres Choisies, Arthur Rousseau, Paris 1911, et dans Antoine SAvoye et Frédéric Audren (dir.), Naissance de l'ingénieur social. Les ingénieurs des mines et la science sociale au XIX siècle (Frédéric Le Play et ses élèves), Presses des Mines, Paris, 2008, p. 295-307.

Dubreuil Hyacinthe, Standards. Le travail américain vu par un ouvrier français, Préface de Henry Le Chatelier, Grasset, Paris, 1929.

Haldane John W. C., Civil \& Mechanical Engineering popularly and socially considered, E. F. \& N. Spoon, London, 1887.

Journal officiel de la République française (JORF) du 13 juillet 1934, p. 7058.

Lamirand Georges, Le Rôle social de l'ingénieur. Scènes de la vie d'usine, éd. de la Revue des jeunes, Desclée et Cie, 1932.

Rizzi Bruno, La Bureaucratisation du monde, Paris, B. Rizzi, 1939.

Schumpeter Joseph A., Theorie der Wirtschaftlichen Entwicklung (Théorie de l'évolution économique), Duncker \& Humblot, Berlin, 1911 et 1926.

SociétÉ CENTRALE DES INGÉNIEURS CIVILS, Statuts adoptés en assemblée générale le 13 juillet 1848, article 2, source : gallica.bnf.fr. 


\section{Bibliographie}

Albu Austen, "British attitudes to engineering education: a historical perspective ", dans PavitT Keith (dir.), Technical Innovation and British Economic Performance, Londres, Palgrave Macmillan, 1980, p. 67-87.

Angio Agnès (d'), Schneider et Cie et la naissance de l'ingénierie. Des pratiques internes à l'aventure internationale, 1836-1949, CNRS Éditions, Paris, 2000.

Baudet Jean C., "Situation des ingénieurs en Belgique. Note de synthèse ", Sociétés contemporaines, 6, juin 1991, p. 119-125.

Bensimon Fabrice, " "Latelier du monde”, 1815-1875 ", dans Stéphane LebecQ (dir.), Histoire des îles Britanniques, PUF, Paris, 2013, p. 611-646.

Berner Boel (dir.), Gendered Practices: Feminist Studies of Technology and Society, Departement of Technology and Social Change, Linköping, 1997.

Berner Boel, "L'ingénieur ou le génie du mâle : masculinité et enseignement technique au tournant du xx ${ }^{\mathrm{e}}$ siècle ", Cahiers du Gedisst, n 19, 1997, p. 7-25.

Bertilorenzi Marco, Dubruc Nadine, Passaqui Jean-Philippe (dir.), Henri Fayol. Les multiples facettes d'un manager, Presses des Mines, Paris, 2019.

Bigazzi Duccio, La grande fabbrica. Organizzazione industriale e modello americano alla Fiat dal Lingotto a Mirafiori, Giangiacomo Feltrinelli, Milan, 2000.

BIrck Françoise, "Témoignage. Des écoles d'ingénieurs de Nancy (XIX ${ }^{\mathrm{e}}$ $\mathrm{XXI}^{\mathrm{e}}$ siècles). Histoire d'un programme de recherche ", Quaderns d'Historia de l'Enginyeria, vol. xv, 2016-2017, p. 77-100.

Blond Stéphane, Hilaire-Pérez Liliane, Nègre Valérie, Virol Michèle (dir.), Les Ingénieurs, des intermédiaires? Transmission et coopération à l'épreuve du terrain (Europe, XVt-XVIII siècle), PUM, Toulouse, coll. Histoire et Techniques, 2020.

Boisard Pierre, Didry Claude, Younès Dima (dir.), Les travailleurs de l'innovation. De l'entrepreneur aux salariés, PUR, Rennes, 2016.

Boltanski Luc, Les Cadres : la formation d'un groupe social, Minuit, Paris, 1982. Bordes Jean-Louis, Champion Annie et Desabres Pascal (dir), L'Ingénieur entrepreneur. Les centraliens et l'industrie, PUPS, Paris, 2011.

Bouneau Christophe, "Les ingénieurs de production et les techniciens qualifiés de l'industrie électrique en France depuis la fin du XIX ${ }^{\mathrm{e}}$ siècle ", dans Guillaume Pierre (dir.), Regards sur les classes moyennes, XIXe-XIXe siècles, Éd. de la MSH d'Aquitaine, Bordeaux, 1995, p. 17-37.

Bouvier Yves, Connexions électriques. Technologies, hommes et marchés dans les relations entre la Compagnie générale d'électricité et l'État, Peter Lang, Bruxelles, 2016.

Brion René, "La querelle des ingénieurs en Belgique ", dans Grelon André (dir.), Les Ingénieurs de la crise, titre et profession entre les deux guerres, EHESS, Paris, 1986, p. 255-270. 
Brucy Guy et Le Bot Florent, "Les deux rêves du technique. De la mise en ordre à la mise en espace ", Artefact, 3, mars 2015, p. 21-37.

Canel Annie et Zachmann Karin (dir.), "Gaining Access, Crossing Boundaries: Women in Engineering in a Comparative Perspective ", History and Technology, $\mathrm{n}^{\circ} 14,1999 / 1-2$.

CAYET Thomas, Rationaliser le travail, organiser la production. Le Bureau international du travail et la modernisation économique durant l'entre-deux-guerres, PUR, Rennes, 2010.

Chabaud-Rycher Danielle et Gardey Delphine (dir.), L'Engendrement des choses, Archives Contemporaines, Paris, 2002.

Chandler Alfred D. Jr., Strategy and Structure. Chapters in the History of the Industrial Entreprise, MIT Press, Cambridge, Mass., 1962

Charle Christophe, "Le pantouflage en France (vers 1880-vers 1980) ", Annales Économies, sociétés et civilisations, ${ }^{\circ}$ 5, sept.-oct. 1987, p. 1115-1137.

Church Roy, The Rise and Decline of the British Motor Industry, Macmillan, Londres, 1994.

Cohen Yves, "Mais que sont donc ces "techniciens sociaux" ? Peugeot Sochaux 1936-1939", Vie sociale, février-mars 1988, p. 41-55.

Cohen Yves, Organiser à l'aube du taylorisme : la pratique d'Ernest Mattern chez Peugeot, Presses universitaires de Franche-Comté, Besançon, 2001.

Cohen Yves, Le Siècle des chefs. Une histoire transnationale du commandement et de l'autorité (1890-1940), Éditions Amsterdam, Paris, 2013.

Collins Paul et Stratton Michael, British Car Factories from 1896, Veloce Publishing, Godmanstone (Dorset), 1993.

DARD Olivier, "Un homme, un livre : Georges Lamirand et le rôle social de l'ingénieur ", dans Bordes Jean-Louis, Champion Annie, Desabres Pascal (dir.), L'Ingénieur entrepreneur : les centraliens et l'industrie, PUS, Paris, 2011, p. 407-418.

DAVIET Jean-Pierre, Un destin international. La Compagnie de Saint-Gobain de 1830 à 1939, Archives contemporaines, Paris, 1988.

DAY R. Charles, Les Écoles d'Arts et Métiers, l'enseignement technique en France, $X I X^{e}-X X^{e}$ siècles, Belin, Paris, 1991.

Derouet Antoine et PAYE Simon (dir.), Les Ingénieurs, unité, expansion, fragmentation (XIXe et XX siècles), Classiques Garnier, Paris, 2018.

Dewerpe Alain, Les Mondes de l'industrie. L'Ansaldo, un capitalisme à l'italienne, EHESS, Paris, 2017.

Dhombres Jean (dir.), La Bretagne des savants et des ingénieurs, 1825-1900, Éd. Ouest France, Rennes, 1994.

Dosse François, Le Pari biographique. Écrire une vie, La Découverte, Paris, 2005. 
Dumons Bruno et Le Bot Florent, "Catholicisme social ", dans Daumas JeanClaude et al. (dir.), Dictionnaire historique des patrons français, Flammarion, Paris, 2010, p. 1013-1017.

EDgerton David, Quoi de neuf: sur le rôle des techniques dans l'histoire globale, Seuil, Paris, 2013.

Емттоz Gérard, "Ingénieurs et techniciens pour les industries de la chimie au XIX ${ }^{e}$ siècle : un aperçu des formations ", dans Pierre LAMARd et Nicolas Sтолкорғ (dir.), L'Industrie chimique en question, Picard, Paris, 2010, p. 15-27.

Emptoz Gérard, Fauque Danielle et BReYsse Jacques (dir.), Entre reconstruction et mutations, les industries de la chimie entre les deux guerres, EDP Sciences, Les Ulis, 2018.

Ferguson Hugh et Chrimes Mike, The Civil Engineers. The Story of the Institution of Civil Engineers and the People Who Made It, Thomas Telford Ltd, Londres, 2011.

Figueiredo Antonio Dias de et Cunha Paulo Rupino da, "Action Research and Design in Information Systems: Two Faces of a Single Coin », dans Kock Ned (dir.), Information Systems Action Research: An Applied View of Emerging Concepts and Methods, Springer, New York, 2007, p. 61-96.

Figueiredo Antonio Dias de, "De la nature historique des pratiques d'ingénierie ", Revue d'anthropologie des connaissances, $\mathrm{n}^{\circ}$ 8, 2014/2, p. 245-278.

Fox Robert et Guagnini Anna, Laboratories, Workshops, and Sites: Concepts and Practices of Research in Industrial Europe: 1800-1914, University of California Press, Berkeley, 1999.

Fridenson Patrick, "Les ingénieurs et les cadres de l'automobile en France au $\mathrm{xx}^{\mathrm{e}}$ siècle ", dans Joutard Philippe et Lecuire Jean (dir.), Histoire sociale, sensibilités collectives et mentalités, PUF, Paris, 1985, p. 431-448.

Fridenson Patrick, "Les cadres et l'organisation de l'entreprise : Renault, 1945-1985 ", dans Jacqueline Costa-Lascoux, Geneviève Dreyfus-Armand et Émile Temime (dir.), Renault sur Seine. Hommes, lieux et mémoires, La Découverte, Paris, 2007, p. 113-133.

Garçon Anne-Françoise, Entre l'État et l'usine, l'École des Mines de Saint-Étienne au XIX siècle, PUR, Rennes, 2004.

Garçon Anne-Françoise, L'Imaginaire et la pensée technique. Une approche historique, $X V V^{e}-X X^{e}$ siècle, Classiques Garnier, Paris, 2012.

Gardey Delphine, "Histoire de pionnières ", Travail, Genre et Sociétés, n 4, 2000/2, p. 29-34.

GARDEY Delphine, "La part de l'ombre ou celles lumières ? Les sciences et la recherche au risque du genre ", Travail, genre et sociétés, n 14, 2005/2, p. 29-47.

GARDEY Delphine et Löwy Ilana (dir.), L'Invention du naturel. Les sciences et la fabrication du féminin et du masculin, Éd. des archives contemporaines, Paris, 2002. 
Gouzévitch Irina, Cardoso de Matos Ana, Fontanon Claudine et al. (dir.), "Ingénieurs, professions techniques et société ( $\mathrm{XVIII}^{\mathrm{e}}-\mathrm{XX}^{\mathrm{e}}$ siècles). Hommage à André Grelon ", Quaderns d'Història de l'Enginyeria, vol. xv (nº spécial), 2016-2017.

Grelon André (dir.), Les Ingénieurs de la crise, titre et profession entre les deux guerres, EHESS, Paris, 1986.

Grelon André, «Marie-Louise Paris et les débuts de l'École Polytechnique féminine (1925-1945) ", Bulletin d'histoire de l'électricité, n 19-20, juin-décembre 1992.

Grelon André, "Formation et carrière des ingénieurs en France (1880-1939) ", dans Louis Bergeron et Patrice Bourdelais (dir.), La France n'est-elle pas douée pour lindustrie?, Belin, Paris, 1998, p. 231-274.

Grelon André et Birck Françoise (dir.), Des ingénieurs pour la Lorraine, XIX'XXe siècles, Éditions Serpenoise, Nancy, 1998.

Grelon André et Birck Françoise (dir.), Un siècle de formation des ingénieurs électriciens. Ancrage local et dynamique européenne. L'exemple de Nancy, Éditions de la MSH, Paris, 2006.

GRISET Pascal (dir.), Les Ingénieurs des télécommunications dans la France contemporaine. Réseaux, innovation et territoires (XIX'-XXe siècles), IGPDE, CHEFF, Paris, 2013.

Henry Odile, Les Guérisseurs de l'économie. Ingénieurs-conseils en quête de pouvoir, CNRS éd., Paris, 2012.

Hobsbawm Eric et Ranger Terence (dir.), L'Invention de la tradition, éd. Amsterdam, Paris, 2006 (anglais, 1983).

Hugot-Piron Nathalie, Les "cadres âgés ". Histoire d'une catégorie de chômeurs, PUR, Rennes, 2014.

JaCQuin François, Les Cadres de lindustrie et du commerce en France, Armand Colin, Paris, 1955.

JaRRIGE François, Technocritiques. Du refus des machines à la contestation des technosciences, La Découverte, Paris, 2014.

Коцвоом Ingo, « Patronat et cadres : la contribution patronale à la formation du groupe des cadres (1936-1938) ", Le Mouvement social, oct.-déc. 1982, n 121, p. 71-95.

KöNIG Wolfgang, "Education and Social Standing German Engineers, 18701930 », Quaderns d'Historia de l'Enginyeria, vol. xv, 2016-2017, p. 113-121.

Kunr-Korolev Corinna et Schlinkert Dirk (dir.), Towards Mobility. Varieties of Automobilism in East and West, Corporate History Department of Volkswagen Aktiengesellschaft, Wolfsburg, 2009.

LaCAITA Carlo G., "Les ingénieurs et l'organisation des études d'ingénieurs en Italie de l'unification politique à la Seconde guerre mondiale ", dans André 
GrELON (dir.), Les Ingénieurs de la crise, titre et profession entre les deux guerres, EHESS, Paris, 1986, p. 309-323.

Lamard Pierre et Stoskopf Nicolas (dir.), L'Industrie chimique en question, Picard, Paris, 2010.

Le Bot Florent, "Capitaines d'industrie ", dans Pierre-Marie Chauvin, Michel Grossetti et Pierre-Paul Zalio (dir.), Dictionnaire sociologique de l'entrepreneuriat, Presses de Sciences Po, Paris, 2015, p. 85-100.

LE Boт Florent, «Enquête sur le céramiste en ingénieur. L’École de céramique de Sèvres instrument de la reconfiguration de l'industrie, 1870-1940 », dans Pierre BoisArd, Claude Didry et Dima Younès (dir.), Les Travailleurs de l'innovation. De l'entrepreneur aux salariés, PUR, Rennes, 2016, p. 85-110.

LE Bot Florent, "De la mondialisation aux mondes de production. Quand les Hommes écrivent leur histoire dans un territoire ", dans Bessy Christian et DidRY Claude (dir.), Robert Salais et l'Économie des conventions : un engagement réflexif, Presses universitaires du Septentrion, Villeneuve d'Ascq, à paraître, 2021.

LE Bot Florent, "Les laboratoires d'essais à l'articulation des écoles techniques supérieures, des entreprises, des territoires et de l'État, 1880-1960 », recherche pour HDR en cours.

LÉCuyer Christophe, "Manager l'innovation », dans Pestre Dominique (dir.), Histoire des sciences et des savoirs, 3-Le siècle des technosciences, Seuil, Paris, 2015, p. 423-439.

LESPINET-MOREt Isabelle, L'Office du travail, La République et la réforme sociale 1891-1914, PUR, Rennes, 2006.

Lewchuk Wayne A., American Technology and the British Vehicule Industry, Cambridge University Press, Cambridge, 1987.

Llopart Michael, "Entre l'État, l'usine et le marché : le "Problème de l'Azote" en France. Retour sur les débuts controversés d'une entreprise publique durant l'entre-deux-guerres. L'office national industriel de l'azote (1924-1940) ", thèse d'histoire contemporaine [dir. A. Boscus et J.-M. Olivier], soutenue publiquement et en visioconférence le 26 juin 2020 à l'université de Toulouse 2 Jean-Jaurès.

Locke Robert R., The End of Practical Man: Entrepreneurship and Higher Education in Germany, France, and Great Britain. 1880-1940, Jai Press, Greenwich, 1984.

Ludwig Karl-Heinz, Technik und Ingenieure im Dritten Reich, Droste, Düsseldorf, 1974.

LuminEAu Léonor, «À compétences égales, les recruteurs choisissent aujourd'hui des femmes ", Le Monde, 20 juin 2011.

Lusa Monforte Guillermo, "L'enseignement industriel pendant la première phase de l'industrialisation espagnole : l'École d'ingénieurs de Barcelone ", dans 
Irina Gouzévitch, André Grelon, Anousheh Karvar (dir.), La Formation des ingénieurs en perspective. Modèle de référence et réseaux de médiation, XVIIt$X X^{e}$ siècles, PUR, Rennes, 2004, p. 35-52.

Lusa Monforte Guillermo, "L'École d'ingénieur de Barcelone et l'électrification dans les années 1875-1899 en Espagne ", dans André Grelon et Françoise Birck (dir.), Un siècle de formation des ingénieurs électriciens. Ancrage local et dynamique européenne. L'exemple de Nancy, Éd. de la MSH, Paris, 2006, p. 405-420.

Macleod Christine, Heroes of Invention. Technology, Liberalism, and British Identity 1750-1914, Cambridge University Press, Cambridge, 2007.

Marnot Bruno, Les Ingénieurs au Parlement, sous la III République, CNRS Éditions, Paris, 2000.

Marry Catherine, Une révolution respectueuse : les fermmes ingénieures, Belin, Paris, 2004.

Marsh Joseph, «Du cercle privé à l'antichambre de l'Université : les associations d'ingénieurs et l'image de marque des ingénieurs britanniques du XVIII ${ }^{\mathrm{e}}$ siècle à nos jours ", dans Grelon André (dir.), Les Ingénieurs de la crise, titre et profession entre les deux guerres, EHESS, Paris, 1986, p. 241-254.

Martykánová Darina, "Les fils du progrès et la civilisation. Les ingénieurs des travaux publics en Espagne aux XVIII ${ }^{\mathrm{e}}$ et $\mathrm{XIX}^{\mathrm{e}}$ siècles ", Quaderns d'historia de l'Enginyeria, vol. x, 2009, p. 251-270.

Michel Alain P., «Le cas Pierre Bézier : Biographie industrielle d’un ingénieur-innovateur (1933-1975) ", mémoire inédit du dossier d'habilitation à diriger des recherches, intitulé "La chaîne, du mécanique au numérique : Histoire technique de fabrications automobiles », 2015, Université d'Évry Paris-Saclay.

Michel Alain P., Pierre Bézier. Une biographie industrielle, Classiques Garnier, Paris, à paraître 2021.

Mommsen Hans et Grieger Manfred, Das Volkswagenswerk und seine Arbeiter im Dritten Reich, Econ Verlag, Düsseldorf, 1996.

Moutet Aimée, "Ingénieurs et rationalisation dans l'industrie française de la Grande Guerre au Front Populaire ", Culture technique, n 12, mars 1984, p. $137-153$.

Moutet Aimée, Les Logiques de l'entreprise. La rationalisation dans l'industrie française de l'entre-deux-guerres, EHESS, Paris, 1997.

Paillat Jacques, "Structure démographique des cadres français ", Sociologie du travail, $\mathrm{n}^{\circ} 3,1960$, p. 246-256.

PAssaqui Jean-Philippe, Les Voyages forment l'ingénieur. Les houillères du CentreMidi de la France, 1851-1873, Classiques Garnier, Paris, 2015.

Passeron Jean-Claude, Revel Jacques (dir.), Penser par cas, EHESS, Paris, 2005. 
Peslouan Geneviève (de), Qui sont les femmes ingénieurs en France?, PUF, Paris, 1974.

Picon Antoine, L'Invention de l'ingénieur moderne. L'École des Ponts et chaussées, 1747-1851, Presses de l'École nationale des Ponts et chaussées, Paris, 1992.

Ribeill Georges, La Révolution ferroviaire. La formation des compagnies de chemin de fer en France (1823-1870), Belin, Paris, 1993.

RoBErt Éric et MORET Roger (dir.), L'Ingénieur moteur de l'innovation, un siècle de formation d'ingénieurs à Grenoble, Institut national polytechnique de Grenoble, Les Éditions des Vignes et l'éditeur, Grenoble, 2001.

Rossiter Margaret, Women Scientists in America. Struggles and Strategies to 1940, John Hopkins University Press, Baltimore and London, 1982.

Schweitzer-Vandecasteele Sylvie, "Comment peut-on être contremaître ? ", dans Lequin Yves et Schweitzer-Vandecasteele Sylvie (dir.), L'Usine et le bureau, itinéraires sociaux et professionnels dans l'entreprise, XIXe et XX' siècle, PUL, Lyon, 1990, p. 93-108.

SHinn Terry, "Des sciences industrielles aux sciences fondamentales. La mutation de l'École supérieure de physique et de chimie (1882-1970) ", Revue française de sociologie, $\mathrm{n}^{\circ} 22,1981$, p. 167-182.

Stevanovic Biljana, "L'histoire de la mixité à l'ex-École Polytechnique féminine, 1969-2000 ", Carrefours de l'éducation, n 17, 2004/1, p. 58-75.

STÜCK Heiner, "L'émancipation des écoles supérieures techniques et la professionnalisation des ingénieurs en Allemagne au XIX ${ }^{\mathrm{e}}$ siècle ", dans Grelon André (dir.), Les Ingénieurs de la crise, titre et profession entre les deux guerres, EHESS, Paris, 1986, p. 271-289.

ThÉpot André (dir.), L'Ingénieur dans la société française, Éd. ouvrières, Paris, 1985.

THÉPOT André, Les Ingénieurs des mines au XIX siècle. Histoire d'un corps technique d'État, Tome 1, 1810-1914, préf. Jean-Louis Beffa, éd. Eska, Paris, 1998.

VATIN François (dir.), Organisation du travail et économie des entreprises (textes choisis et commentés de Taylor, Amar, Lahy, Le Chatelier, Belot), Éd. de l'organisation, Paris, 1990.

Vaughan Adrian, Brunel: an Engineering Biography, Ian Allan Publishing, Shepperton, 2006.

VÉrIN Hélène, La Gloire des ingénieurs. L'intelligence technique du XVI au XVIII siècle, Albin Michel, Paris, 1993.

WeIss John H., The Making of Technological Man. The Social Origins of French Engineering education, MIT Press, Cambridge (Mass.), 1982. 
Florent Le Bot et Alain P. Michel

\section{Les auteurs}

Florent Le Bot est enseignant-chercheur en Histoire contemporaine à l'Université d'Évry Paris-Saclay, IDHES (UMR CNRS 8533). Ses recherches portent sur l'histoire économique et industrielle de l'Europe de l'Ouest aux $x \mathrm{x}^{\mathrm{e}}$ et $\mathrm{xx}$ siècles. Il a notamment publié La fabrique réactionnaire. Antisémitisme, corporatisme et spoliations dans le cuir, Paris, Presses de Sciences Po, 2007 ; Histoire économique de Vichy. L'État, les hommes, les entreprises (avec F. Grenard et C. Perrin), Paris, Perrin, 2017 et L'échelle des régulations politiques, XVIII -XXI siècles. L'histoire et les sciences sociales aux prises avec les normes, les acteurs et les institutions (avec V. Albe et J. Commaille, dir.), Villeneuve-d’Ascq, Presses universitaires du Septentrion, 2019. Contact : florent.lebot@univ-evry.fr

Alain P. Michel est professeur d'histoire contemporaine à l'Université d'Évry ParisSaclay. Il est directeur de l'IDHES-Évry (UMR CNRS 8533). Il est à la fois historien des techniques industrielles et spécialiste des sources visuelles. Il a récemment publié « Out of Control. Telephone Network and Management of Business Talks at Renault (1911-1939) », Information \& Culture, 2016, vol. 51, n 4 et « Pierre Bézier et ses machines-transferts Renault (1940-1960). Quand le cinéma ne suffit pas à consacrer un héros de l'innovation industrielle », Technologie et Innovation, 2019, vol. $4, n^{\circ} 4$. Il termine la publication de son HDR Pierre Bézier. Une biographie industrielle à paraître chez Classique Garnier.Contact : alain.michel@univ-evry.fr 